LAWRENCE LIVERMORE N A TION AL LABORATORY

\section{Optical calibration of pressure sensors for high pressures and temperatures}

Alexander F. Goncharov, Eugene Gregoryanz, Joseph M. Zaug, Jonathan C. Crowhurst

October 6, 2004

Journal of Applied Physics 
This document was prepared as an account of work sponsored by an agency of the United States Government. Neither the United States Government nor the University of California nor any of their employees, makes any warranty, express or implied, or assumes any legal liability or responsibility for the accuracy, completeness, or usefulness of any information, apparatus, product, or process disclosed, or represents that its use would not infringe privately owned rights. Reference herein to any specific commercial product, process, or service by trade name, trademark, manufacturer, or otherwise, does not necessarily constitute or imply its endorsement, recommendation, or favoring by the United States Government or the University of California. The views and opinions of authors expressed herein do not necessarily state or reflect those of the United States Government or the University of California, and shall not be used for advertising or product endorsement purposes. 
Optical calibration of pressure sensors for high pressures and temperatures

Alexander F. Goncharov (a), Eugene Gregoryanz (b), Joseph M. Zaug (a), Jonathan C. Crowhurst (a)

(a) Lawrence Livermore National Laboratory, University of California

(b) Geophysical Laboratory, Carnegie Institution of Washington

We present the results of Raman scattering measurements of diamond $\left({ }^{12} \mathrm{C}\right)$ and of cubic boron nitride $(\mathrm{cBN})$, and fluorescence measurements of ruby, Sm:YAG, and $\mathrm{SrB}_{4} \mathrm{O}_{7}: \mathrm{Sm}^{2+}$ in the diamond anvil cell (DAC) at high pressures and temperatures. These measurements were accompanied by synchrotron $\mathrm{x}$-ray diffraction measurements on gold. We have extended the room-temperature calibration of Sm:YAG in a quasihydrostatic regime up to $100 \mathrm{GPa}$. The ruby scale is shown to systematically underestimate pressure at high pressures and temperatures compared with all other sensors. On this basis, we propose a new high-temperature ruby pressure scale that should be valid to at least $100 \mathrm{GPa}$ and $850 \mathrm{~K}$. 
Historically, the accurate determination of pressure at high temperature and ultrahigh pressure has been extremely difficult. In fact, the lack of a general pressure scale nullifies, to a significant extent, the great innovations that have been made in recent years in DAC experimental techniques [1]. Now, more than ever a scale is required whose accuracy is comparable with that of the experimental data. Since pressure in the DAC is dependent on temperature (due to thermal pressure and also to changes in the properties of the materials that constitute the DAC) such a scale requires quantitative, and separate measurements of pressure and temperature.

The room-temperature high-pressure scale was established some 25 years ago. It was based on a comparison between the lattice constants of several simple metals measured under static conditions to the corresponding equations of state determined in dynamic compression experiments [2]. The necessary temperature correction of the lattice constants was performed on the basis of Mie-Gruneisen theory. This high-pressure scale (called the ruby scale after the name of the convenient secondary fluorescence gauge) is claimed to be accurate to within $5 \%$ to $100 \mathrm{GPa}$. Use of this scale under quasihydrostatic conditions [3] is believed to be still more accurate. An accurate primary pressure scale $(\mathrm{MgO})$ has also been developed by combining equation-of-state data with elasticity measurements [4]. Although the method of using a metal primary standard [2] can be applied at any temperature (with an appropriate temperature correction), in practice this is rarely used except in combination with x-ray diffraction studies for which a suitable 
intense source is available (e.g., a synchrotron source). This type of measurement is difficult to combine with other experiments (e.g., optical). Optical measurements of ruby (and other secondary optical sensors) remain the preferred means of determining pressure in table-top experiments.

The ruby fluorescence technique does however present some significant drawbacks (see, e.g. Ref. [5] and references therein). The line width of the (typically) measured fluorescence component (the R1 line) is very sensitive to non-hydrostatic stress and also to temperature. Since the R1 line is one member of a doublet, such conditions result in the overlapping of the two lines and the formation of a broad asymmetrical band. The accuracy of the pressure measurement is then significantly reduced. Furthermore, the signal-to-background ratio of the fluorescence rapidly decreases with temperature and the line is hardly measurable above $700 \mathrm{~K}$. A similar effect is observed in non-hydrostatic environments, making the measurement difficult above $100 \mathrm{GPa}$. Also, since the wavelength of the R1 line depends relatively strongly on temperature the accuracy of the pressure determination is limited by the accuracy of the temperature measurements. An uncertainty of $5 \mathrm{~K}$, for example, leads to an uncertainty of about $0.1 \mathrm{GPa}$ in pressure (at pressures close to ambient). At high pressures the ruby fluorescence shift with temperature has not been measured (but is believed to be substantial), so the pressure measurements are uncertain even in the case of precise determination of temperature.

Materials doped by rare-earth ions offer better performances as fluorescence gauges because they produce very sharp lines which are believed to be less sensitive to 
temperature compared to the $3 \mathrm{~d}$ electrons in $\mathrm{Cr}^{3+}$ (i.e. the dopant in $\mathrm{Al}_{2} \mathrm{O}_{3}$ that produces ruby). For example, the Y band of Sm:YAG has been found attractive because its frequency shift with temperature is negligible up to $820 \mathrm{~K}$ and that with pressure is comparable to ruby $(0.3360 \mathrm{~nm} / \mathrm{GPa})[6,7]$. However, this $\mathrm{Y}$ band is composed of many peaks, which, like ruby, strongly overlap at high pressure and/or high temperature. Furthermore, the relative intensity of the different lines drastically changes under pressure, which makes the pressure measurement difficult and often unreliable.

Another material with excellent fluorescence properties is $\mathrm{SrB}_{4} \mathrm{O}_{7}: \mathrm{Sm}^{2+}[8,9]$. The ${ }^{7} D_{0^{-}}$ ${ }^{5} F_{0}$ fluorescence line at $685 \mathrm{~nm}(0-0$ transition $)$ of this material has a pressure dependence that is comparable to that of other sensors, and also a very weak temperature dependence. It is also relatively insensitive to non-hydrostatic stresses [10]. Therefore, it is a very good substitute for ruby for both very high pressure (Mbar) and high temperature measurements (at least to $900 \mathrm{~K}$ ) [10].

The spectral position of the first-order Raman peaks of diamond and cBN have been proposed as high-pressure gauges [11-13] because of the stability of these materials at high pressure and a sufficiently large frequency shift with pressure. Theoretical calculations [11] and simple empirical relations $[12,13]$ appear to be sufficiently accurate to predict the frequency change of the zone-center optical phonons with pressure and temperature. Recent combined Raman and x-ray studies to $140 \mathrm{GPa}$ [14] and density functional calculations [15] of the diamond equation of state and the pressure-induced Raman shift can serve as a basis of independent high-pressure calibration in the Mbar 
pressure range. Unlike fluorescence gauges, diamond and cBN sensors are very stable at elevated temperatures even in chemically active environment. The use of these materials as pressure gauges at high temperature requires an accurate calibration function, which is known to contain coupled pressure and temperature terms [16].

In this work we review critically the use of several of the most popular high-pressure sensors at high temperature and suggest a modified ruby calibration scale for high temperature measurements. Specifically, we show that the use of the temperature correction of the ruby fluorescence wavelength established at ambient pressure $[5,10]$ leads to significant inconsistencies with the results obtained by using other standards at high pressures (above $15 \mathrm{GPa}$ ). On the contrary, the other commonly used fluorescence gauges $\left(\mathrm{SrB}_{4} \mathrm{O}_{7}: \mathrm{Sm}^{2+}\right.$ and $\left.\mathrm{Sm}: \mathrm{YAG}\right)$, which require only a negligible temperature correction at low pressure show consistent results at high pressures (up to $100 \mathrm{GPa}$ ) when used without a temperature correction. Raman shifts of cBN and diamond show consistent results in the 30-40 GPa range. Based on the results of measurements of different pressure gauges, we propose a pressure calibration for ruby at high temperature, which contains coupled pressure and temperature terms.

We performed a series of optical experiments at high pressures and temperatures (combined with x-ray diffraction in some cases) in order to establish correlations between different sensors. The external resistively heated DAC have been used, equipped with two heaters and thermocouples. The temperature was measured to within $\pm 1 \mathrm{~K}$ below 600 $\mathrm{K}$ and $\pm 5 \mathrm{~K}$ above $600 \mathrm{~K}$. Ruby, $\mathrm{SrB}_{4} \mathrm{O}_{7}: \mathrm{Sm}^{2+}, \mathrm{Sm}$ :YAG fluorescence, ${ }^{12} \mathrm{C},{ }^{13} \mathrm{C}$ diamond 
and cBN Raman and Au X-ray sensors have been studied. Solid nitrogen and hydrogen $[17,18]$ typically served as a pressure-transmitting media in order to minimize shear stresses in the gauges. The optical experiments have been performed with the Raman system that has been described previously (see Ref. [19] and references therein). The combined optical and x-ray measurements were performed using a custom built portable Raman/ fluorescence microscope, incorporating a laser source and a spectrum analyzer (spectrograph with 1- $\mathrm{N}_{2}$ cooled CCD detector) by optical fibers. Powder diffraction measurements were performed using monochromatic synchrotron radiation. Diffraction patterns were collected by CCD or image plate detectors. These measurements were performed at the Stanford Synchrotron Radiation Laboratory, and at the Advanced Photon Source (HPCAT).

To compare the results obtained for various sensors, we have assumed the following calibration dependences. The ruby pressure scale at room temperature [3] was combined with the pressure-independent temperature correction [10]. The $\mathrm{SrB}_{4} \mathrm{O}_{7}: \mathrm{Sm}^{2+}$ scale that we used was the improved calibration reported in Ref. [10]. We used our own Sm:YAG calibration (see below), based on the position of the Y2 band [6,7], because we found that the calibration reported in Ref. [7] to $20 \mathrm{GPa}$ underestimates pressure beyond this limit. The equation of state of gold has been revised recently [20], based on the inversion of quasi-hydrostatic compression and shock wave data using the Mie-Grüneisen relation and the Birch-Murnaghan-Debye equation. We determined the corresponding pressure accordingly. 
The positions of the first-order Raman bands in cBN were used for pressure determination based on concurrent Raman and x-ray studies [12,13]. Temperature shift (determined at ambient pressure [21]) was assumed to be pressure independent. This determination is in agreement with the calibration proposed in a recent study to $20 \mathrm{GPa}$ [16]. In the case of diamond we also assumed independent pressure [12] and temperature $[22,23]$ terms. In the pressure-temperature range of this work (30 GPa, $700 \mathrm{~K})$ introduction of the cross terms [16] would result in a negligible correction.

We performed a series of experiments with the externally heated DAC [25], using ruby and Sm:YAG loaded in the same high-pressure cavity with compressed hydrogen and nitrogen as a pressure medium. Fig. 1 shows the pressure dependence of the wavelength of the most pronounced fluorescence bands of Sm:YAG. We find that most of the bands become very broad and weak above $60 \mathrm{GPa}$, and only the Y2 band could be easily traced to $100 \mathrm{GPa}$. The Y4 peak (Y6 in notation of Ref. [7]), reported in Ref. [24] at pressures beyond $100 \mathrm{GPa}$ was masked in our experiments by the Raman band corresponding to the hydrogen vibron. The pressure was determined according to the ruby scale [3] and consistent with measurements of the hydrogen vibron frequency [26]. The pressure dependence of the Y2 band is linear up to $100 \mathrm{GPa}$ (Fig. 1) and can be used as an alternative calibration at high temperatures. The best fit to the experimental data gives the following formula for determination of $\mathrm{P}$ using the $\mathrm{Y} 2$ line of the $\mathrm{YAG}$ sensor:

$\mathrm{P}(\mathrm{GPa})=3.29 *(\lambda(\mathrm{nm})-615.6) \quad(\mathrm{Y} 2)$.

In the pressure range to $60 \mathrm{GPa}$, the $\mathrm{Y} 1, \mathrm{Y} 3$ and $\mathrm{Y} 6$ lines can also be used for the pressure measurements using the following equations: 
$\mathrm{P}(\mathrm{GPa})=3.15^{*}(\lambda(\mathrm{nm})-617.7) \quad(\mathrm{Y} 1)$,

$\mathrm{P}(\mathrm{GPa})=3.08 *(\lambda(\mathrm{nm})-610.7) \quad(\mathrm{Y} 3)$,

$\mathrm{P}(\mathrm{GPa})=6.37 *(\lambda(\mathrm{nm})-603.1) \quad(\mathrm{Y} 6)$.

At ambient pressure, the spectral position of the $\mathrm{Y}$ lines is almost temperature independent $[6,7]$. Assuming that this observation holds under pressure, the Sm:YAG sensor can be used for independent estimation of pressure at high temperatures.

Fig. 2 shows the results of two temperature runs to $700 \mathrm{~K}$ Pressure was drifting with temperature as indicated by both gauges. Assuming that the slope of the temperature dependence of the ruby R1-line is pressure independent, one can see that the discrepancy between ruby and Sm:YAG measurements increases with temperature. Assuming that the temperature dependence of the R1 ruby fluorescence becomes more moderate with pressure (see below), we are able to match the pressures indicated by the two gauges at high temperatures.

The second series of experiments included a larger number of optical gauges and also an x-ray diffraction-based gauge (gold), enclosed in the same cavity. Nitrogen was used as the pressure medium. Fig. 3 shows the results of selected measurements for different pressure sensors compared with the ruby scale in the pressure range of 30-40 GPa. They show systematic deviations from the ruby scale that increase with temperature. In addition, the pressure indicated by diamond and $\mathrm{cBN}$ is larger even at room temperature, which suggests that the ruby scale may underestimate pressure $[12,15,27]$. This 
discrepancy increases with pressure and it becomes larger than the experimental uncertainty above $30 \mathrm{GPa}$.

We are not aware of any data, which would indicate that the spectral position of the Sm:YAG and $\mathrm{SrB}_{4} \mathrm{O}_{7}: \mathrm{Sm}^{2+}$ sensors depend on temperature substantially. On the contrary, the spectral position of the R1-R2 ruby lines is strongly temperature dependent at ambient pressure $[5,10]$, and there is an indication that the slope of this dependence may be pressure dependent (Ref. [28], based on measurements below $15 \mathrm{GPa}$ ). In attempt to obtain consistent data, we introduce the pressure-dependent temperature coefficient for the ruby fluorescence R1 band in a simple linear form:

$\Delta \lambda / \Delta \mathrm{T}(\mathrm{nm} / \mathrm{K})=0.0073-a^{*} \mathrm{P}$,

where $a=3.5(4)^{*} 10^{-5}\left(\mathrm{~nm}^{*} \mathrm{~K}^{-1} \mathrm{GPa}^{-1}\right)$ determines the variation of this coefficient with pressure.

A more convenient equation, which determines pressure at high temperature (295-800 K) from the spectral position of the R1 ruby fluorescence line, is the following $\mathrm{P}=(\mathrm{B}(\mathrm{T}) / \mathrm{B} 1(\mathrm{~T}))^{*}\left(\left(\lambda(\mathrm{T}) / \lambda_{0}(\mathrm{~T})\right)^{\mathrm{B} 1(\mathrm{~T})}-1\right)$,

where $\lambda(\mathrm{T})$ - is the measured ruby $\mathrm{R} 1$ line wavelength in $\mathrm{nm}, \mathrm{B}(\mathrm{T})=1849.3(\mathrm{GPa})+0.182 * \mathrm{~T}$ $(\mathrm{K}), \mathrm{B} 1(\mathrm{~T})=7.13+0.00178 * \mathrm{~T}(\mathrm{~K}), \lambda_{0}(\mathrm{~T})=692.24+0.00702 * \mathrm{~T}(\mathrm{~K})$. It allows the determination of pressure directly without calculating a reference $300 \mathrm{~K}$ pressure, which is necessary for using formula (1). 
We can now reconcile the data obtained with different pressure sensors in all our experiments (see, for example, Fig. 1). The combined results are presented in Fig. 4. As seen from this figure, the temperature dependence of the R1 ruby fluorescence becomes less pronounced at high pressure. There is a small deviation in the results obtained from different sensors. These discrepancies may be due to relatively large experimental uncertainties, because every experimental point corresponds to two pressure measurements (i.e., ruby and the other gauge). They may also indicate that some of the assumptions about the temperature dependence of the high-pressure gauges made above are not quite accurate. Nevertheless, despite possible uncertainties in the hightemperature behavior of these gauges, a firm conclusion about the ruby scale at high pressures and temperatures can be made: it underestimates pressure and must be corrected. The proposed temperature correction of the ruby scale (equations $(1,2)$ ) suggests that it does not almost require any temperature correction at Megabar pressuresa result with important implications for experiments at extreme conditions. At low temperatures the ruby R1 wavelength is also temperature dependent (see e.g. Ref. [29]), and our study suggests that this dependence is less steep at 100-200 GPa, as was previously believed, but this statement requires an additional experimental confirmation. Also, we believe that the change of the sign of the temperature dependence at about 200 GPa implied by Equation (1) is an artifact of our simplistic model. The proposed temperature correction of the ruby scale was assumed in measurements of the melting curve of hydrogen, and produced a self-consistent data set up to $150 \mathrm{GPa}$ [18]. Use of other pressure gauges at such extreme conditions still requires experimental confirmation, 
although fluorescence gauges $\left(\mathrm{SrB}_{4} \mathrm{O}_{7}: \mathrm{Sm}^{2+}\right.$ and $\left.\mathrm{Sm}: \mathrm{YAG}\right)$ do seem to require any temperature corrections.

We thank D. Häusermann and M. Somayazulu for excellent support at HPCAT (APS). We also thank C. Young for preparation of $\mathrm{SrB}_{4} \mathrm{O}_{7}: \mathrm{Sm}^{2+}$ material and D. Hansen for technical help. Use of the HPCAT facility was supported by DOE-BES, DOE-NNSA (CDAC), NSF, DOD -TACOM, and the W.M. Keck Foundation. Portions of this research were carried out at the Stanford Radiation Laboratory, a national user facility operated on behalf of the U.S. Department of Energy, Office of Basic Energy Science. This work was performed under the auspices of the U. S. Department of Energy by the University of California, Lawrence Livermore National Laboratory under Contract No.W-7405-Eng-48. 
Bibliography.

1. R. J. Hemley, H. K. Mao, In: Encycl. of Appl. Phys., ed. G. L. Trigg,

VCH Publishers, New York, 18, 555 (1997).

2. H. K. Mao, P. M. Bell, J. W. Shaner, and D. J. Steinberg, J. Appl. Phys., 49, 3276 (1978).

3. H. Mao, J. Xu, and P. Bell, J. Geophys. Res. 91, 4673 (1986).

4. C.-S. Zha, H.-K. Mao, and R. J. Hemley, PNAS, 97, 13495 (2000).

5. D. D. Ragan, R. Gustavsen, and D. Schiferl, J. Appl. Phys., 72, 5539 (1992).

6. N. Hess, D. Schiferl, J. Appl. Phys., 68, 1953 (1990).

7. Y. Zhao, W. Barvosa-Carter, S. D. Theiss, S. Mitha, M. J. Aziz, D. Schiferl, J. Appl. Phys., 84, 4049, (1998).

8. A. Lacam and C. Chateau, J. Appl. Phys. 66, 366 (1989).

9. J. Leger, C. Chateau, and A. Lacam, J. Appl. Phys. 68, 2351 (1990).

10. F. Datchi, R. LeToullec, and P. Loubeyre, J. Appl. Phys. 81, 3333 (1997).

11. M. Hanfland, K. Syassen, S. Fahy, S. Louie, and M. Cohen, Phys. Rev. B 31, 6896 (1985).

12. I. V. Aleksandrov, A. F. Goncharov, A. N. Zisman, S. M. Stishov, Sov. Phys. JETP 66, 384 (1987).

13. E. V. Jakovenko, I. V. Aleksandrov, A. F. Goncharov, S. M. Stishov., Sov. Phys. JETP, 68, 1213 (1989).

14. F. Occelli, P. Loubeyre, and R. LeToullec, Nature Material 2, 151 (2003).

15. K. Kunc, I. Loa, and K. Syassen, Phys. Rev. B 68, 094107 (2003).

16. F. Datchi, and B. Canny, Phys. Rev. B. 69, 144106 (2004). 
17. E. Gregoryanz, A. F. Goncharov, R. J. Hemley, H-K. Mao, M. Somayazulu, and G. Shen, Phys. Rev. B 66, 224108 (2002).

18. E. Gregoryanz, A. F. Goncharov, K. Matsuishi, H.-K. Mao, and R. J. Hemley, Phys. Rev. Lett. 90, 175701 (2003).

19. A. F. Goncharov, and V. V. Struzhkin, J. Raman Spectrosc. 34, 532 (2003).

20. S.-H. Shim, T. S. Duffy, K. Takemura, Earth and Planetary Science Letters 203, 729 (2002).

21. H. Herchen, M. A. Cappelli, Phys. Rev. B 47, 14193 (1993).

22. H. Herchen, M. A. Cappelli, Phys. Rev. B 43, 11740 (1991).

23. D. Schiferl, M. Nicol, J. M. Zaug, S. K. Sharma, T. F. Cooney, S.-Y. Wang, T. R. Anthony and J. F. Fleischer, J. Appl. Phys. 82, 3256 (1997).

24. J. Liu and Y. Vohra, J. Appl. Phys. 79, 7978 (1996).

25. Y. Fei, in Mineral Spectroscopy: A Tribute to Roger G. Burns, edited by M. D. Dyar, C. McCammon, and M. W. Schafer, (Geochemical Society, Houston, 1996), p.

26. S. K. Sharma, H.-K. Mao, and P. M. Bell, Phys. Rev. Lett. 44, 886 (1980).

27. W. Holzapfel, J. Appl. Phys. 93, 1813 (2003).

28. S. Rekhi, L. S. Dubrovisky, S. K. Saxena, High Pressure-High Temperature 31, 299 (1999).

29. W. L. Vos and J. A. Schouten, J. Appl. Phys. 69, 6744 (1991). 
Figures.

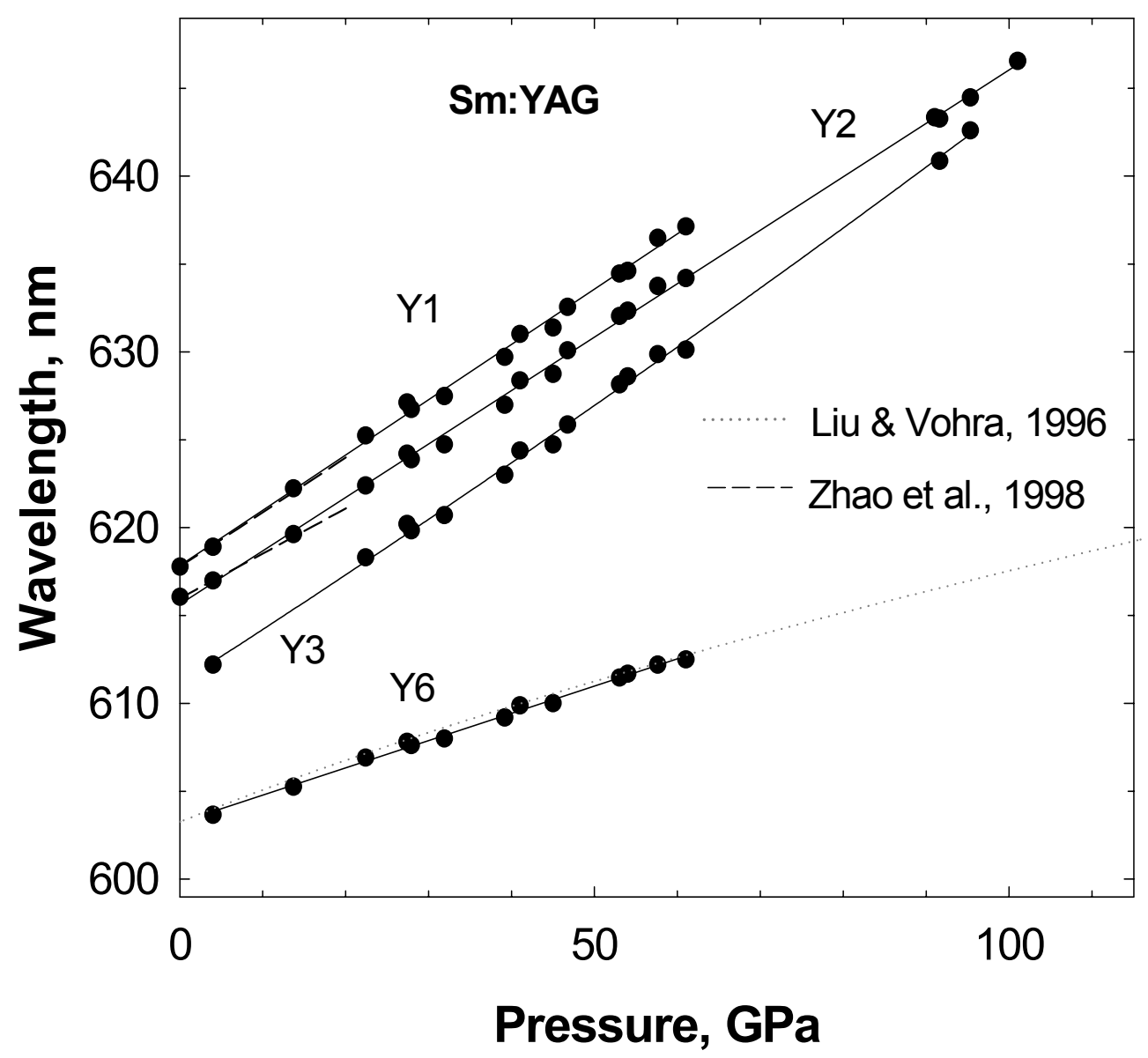

Fig. 1. Pressure dependence of the Sm:YAG fluorescence bands at room temperature.

Data obtained with $\mathrm{N}_{2}$ and $\mathrm{H}_{2}$ transmitting media do not show any systematic deviation from each other. Filled circles and solid lines - our data, dashed line- from Zhao et al. [7], dotted line - from Liu and Vohra [24]. 

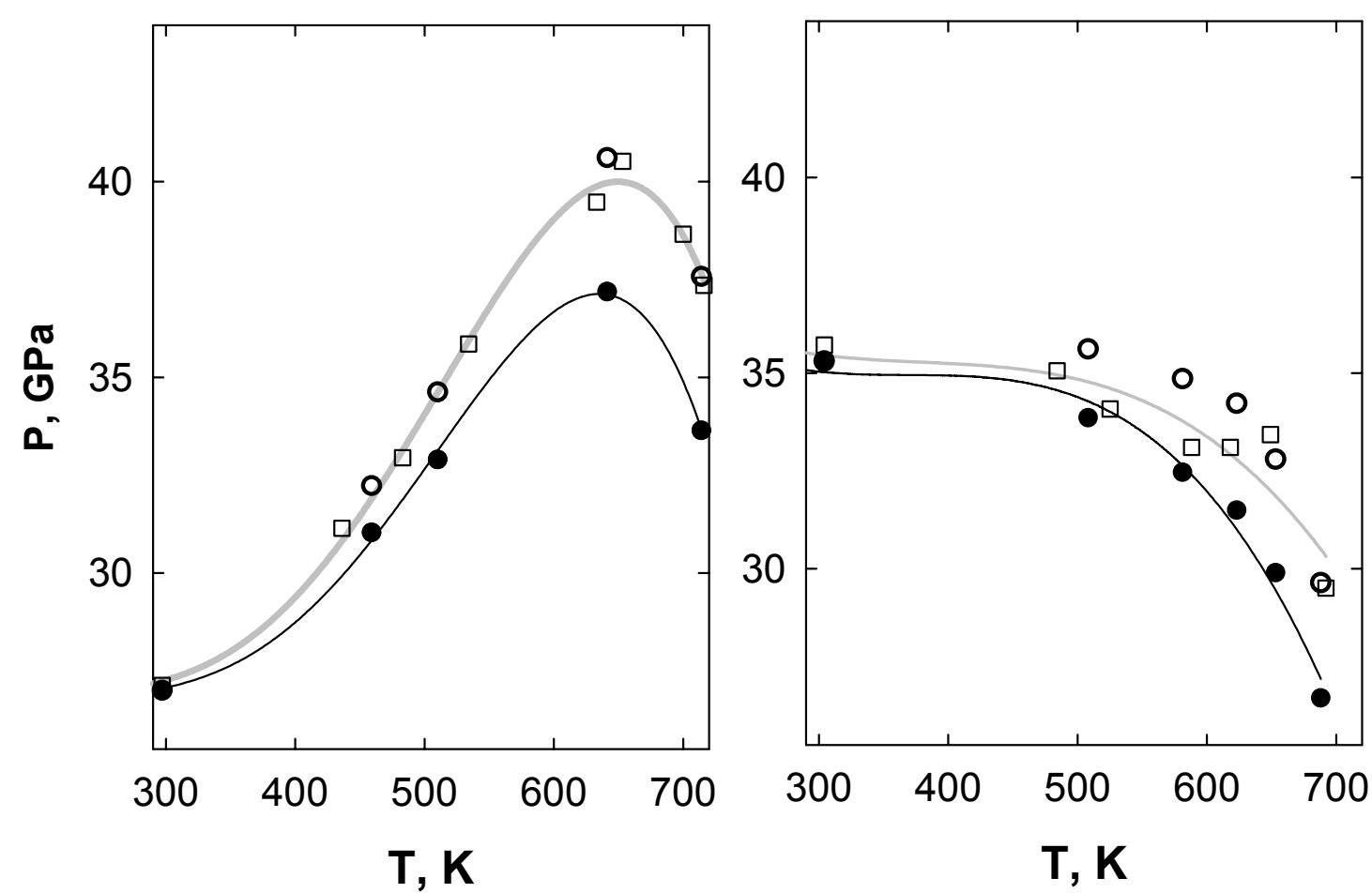

Fig. 2. Pressure variation as a function of temperature in experiments with a hydrogen pressure medium (2 runs, left and right panels) as indicated by Sm:YAG and ruby. Squares - Sm:YAG; filled circles - ruby with pressure-independent, and open circles, with pressure-dependent temperature dependence, respectively (see text). Lines are guides to the eye. 


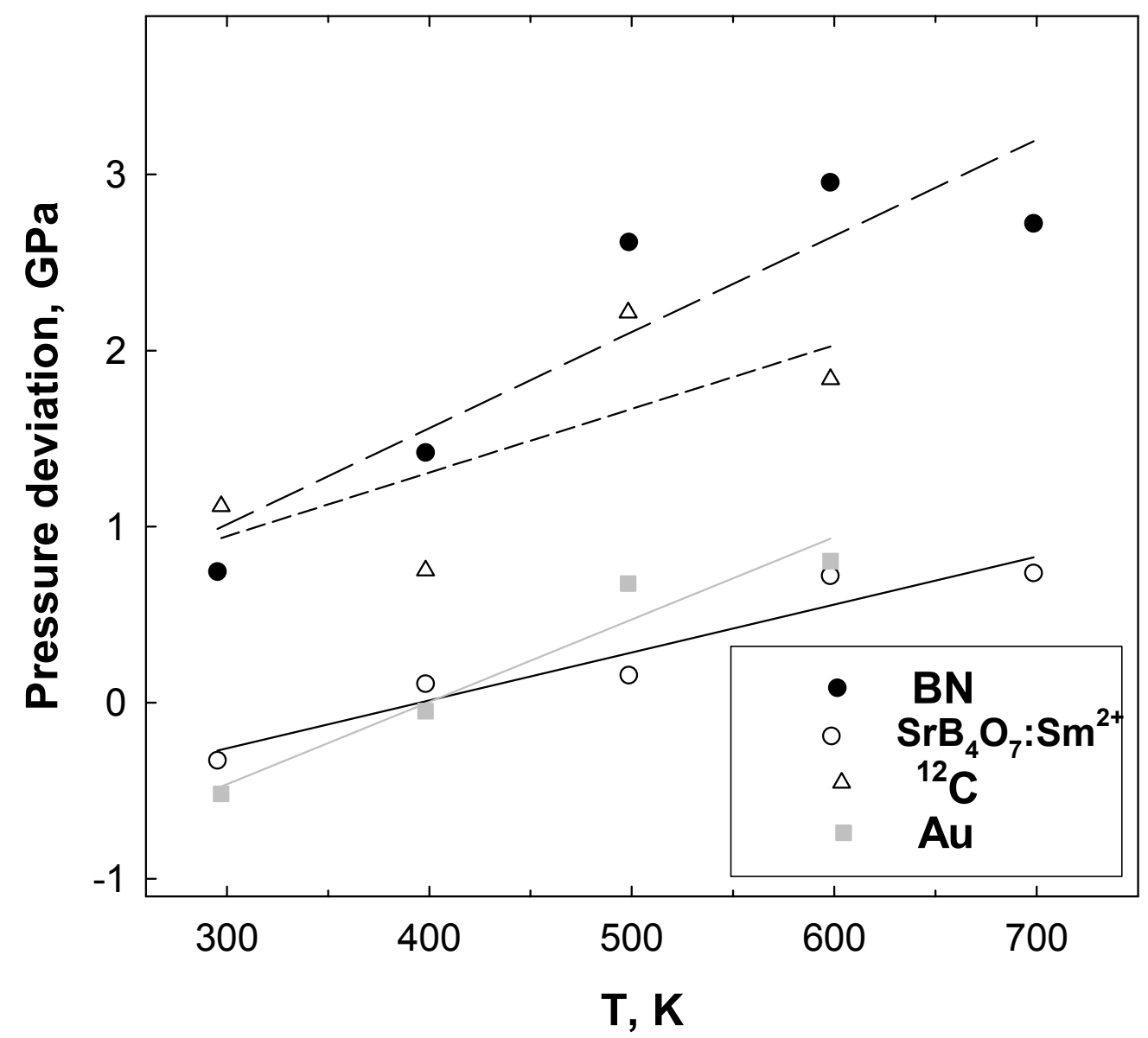

Fig. 3. Temperature dependence of the pressure deviation measured by different gauges with respect to that determined using the conventional ruby scale. Nominal pressure is in the $30-40 \mathrm{GPa}$ range. 


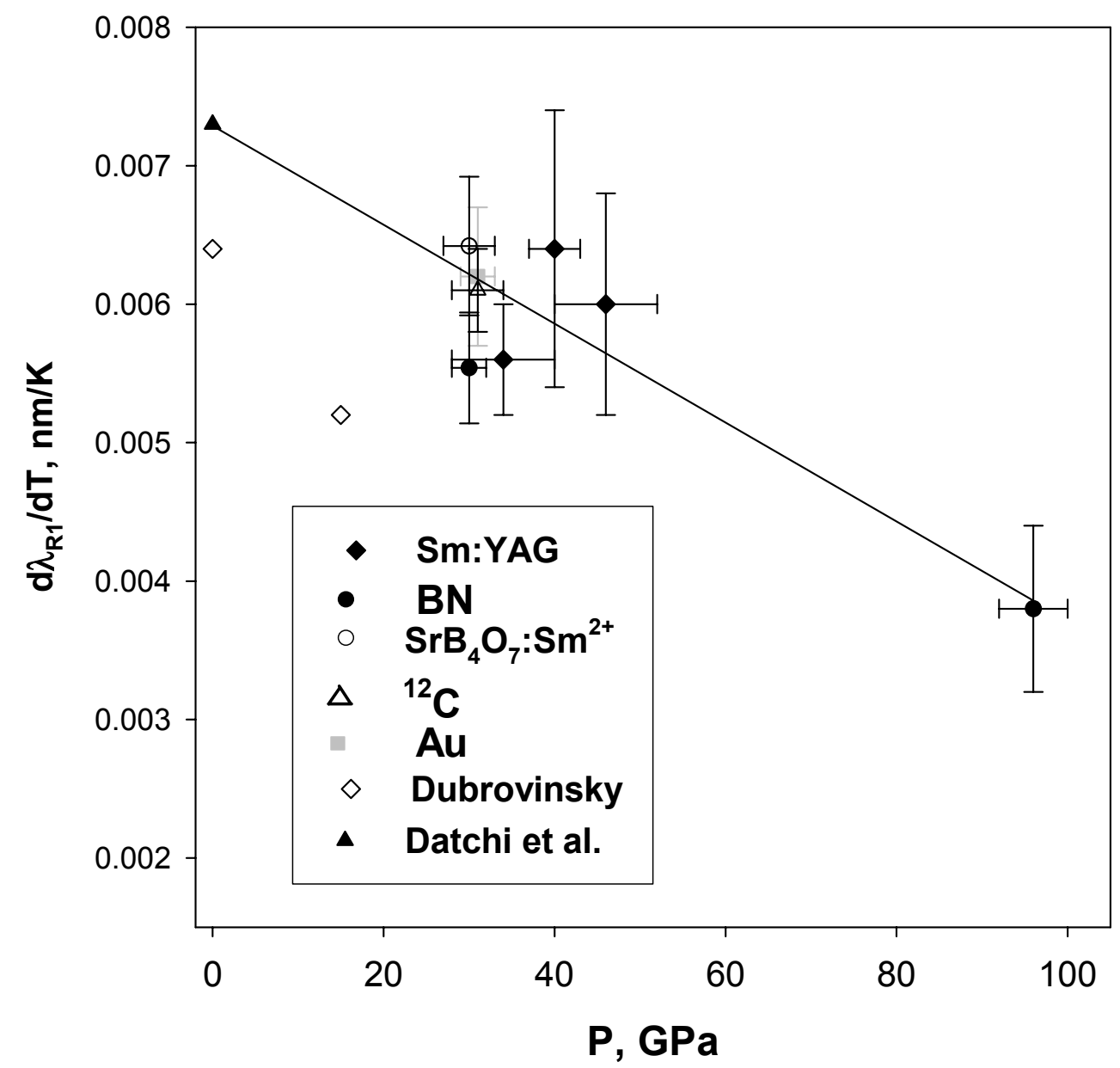

Fig. 4. Pressure dependence of the temperature derivative of the ruby R1 spectral position. The solid line is the best fit to our data determined by using different sensors (different symbols, see legend) and that of Ref. [10] at ambient pressure. Data of Ref. [28] are shown for comparison. 